\title{
A layer model of ethanol partitioning into lipid membranes
}

\author{
David T. Nizza and Klaus Gawrisch
}

Laboratory of Membrane Biochemistry and Biophysics, National Institute on Alcohol Abuse and Alcoholism, NIH, Bethesda, Maryland, U.S.A.

\begin{abstract}
The effect of membrane composition on ethanol partitioning into lipid bilayers was assessed by headspace gas chromatography. A series of model membranes with different compositions have been investigated. Membranes were exposed to a physiological ethanol concentration of 20 $\mathrm{mmol} / \mathrm{l}$. The concentration of membranes was $20 \mathrm{wt} \%$ which roughly corresponds to values found in tissue. Partitioning depended on the chemical nature of polar groups at the lipid/water interface. Compared to phosphatidylcholine, lipids with headgroups containing phosphatidylglycerol, phosphatidylserine, and sphingomyelin showed enhanced partitioning while headgroups containing phosphatidylethanolamine resulted in a lower partition coefficient. The molar partition coefficient was independent of a membrane's hydrophobic volume. This observation is in agreement with our previously published NMR results which showed that ethanol resides almost exclusively within the membrane/water interface. At an ethanol concentration of $20 \mathrm{mmol} / \mathrm{l}$ in water, ethanol concentrations at the lipid/water interface are in the range from 30-15 mmol/l, corresponding to one ethanol molecule per 100-200 lipids.
\end{abstract}

Key words: Ethanol — Lipid bilayer — Partitioning - Headspace gas chromatography

\section{Introduction}

It has been noted for over a century that the anesthetic potency of a particular substance can be correlated to its oil/water partition coefficient, the so-called MeyerOverton rule. A logical extension of this observation is the theory that small organic molecules such as alcohols act upon cells by partitioning into the membrane bilayer causing changes to both bilayer and integral protein structure. Initially, it was assumed that the ability of ethanol to lower lipid order was responsible for ethanol-induced shifts in membrane function (Chin and Goldstein 1977). However, since the 1980's the majority of researchers have assumed that ethanol action is the result of binding to putative hydrophobic pockets in proteins like the binding of a drug to a receptor (Franks and Lieb 1984). Several proteins whose function is influenced by the presence of physiological concentrations of ethanol were identified. Still, evidence for binding motives remains vague and

Correspondence to: Klaus Gawrisch, National Institute on Alcohol Abuse and Alcoholism, NIH, 5625 Fishers Ln., Rm. 3N07, Bethesda, Maryland 20892-9410, U.S.A.

E-mail: gawrisch@helix.nih.gov functional implications of ethanol binding are poorly understood. The quest for understanding the action of ethanol on biopolymers at the level of molecular interactions continues. This includes interaction of ethanol with the lipid matrix.

Measuring the membrane/water partition coefficient of ethanol has proved to be problematic. Much of this stems from the weak binding of ethanol to biological macromolecules, requiring concentrations in the millimolar range for action, as well as the difficulty in dealing with the molecule's inherent volatility. Partition coefficients must be measured directly on lipid bilayers since an isotropic hydrophobic liquid, e.g. an oil, is not an accurate model for the amphipathic, anisotropic cell membrane. When partitioning into the bilayer, ethanol resides predominately at the membrane/water interface (Barry and Gawrisch 1994; Feller et al. 2002). Ethanol's relatively hydrophilic nature causes it to exhibit a relatively lower degree of partitioning into the hydrophobic core of the bilayer, in difference to longer chain alcohols that insert into the hydrophobic core with their hydrophobic chains aligned parallel to the lipid hydrocarbon chains (Westerman et al. 1988).

Ethanol partition coefficients into lipid bilayers have been measured using a variety of methods including radioisotopes 
(Katz and Diamond 1974a), calorimetry (Rowe 1982; Trandum et al. 1999), and vapor pressure (Westh and Trandum 1999). The deviation between reported values is considerable which makes it desirable to measure partitioning with a new and, perhaps, more accurate approach. We used headspace gas chromatography (GC) to measure ethanol vapor pressure above samples. According to Henry's Law, the ethanol vapor pressure in thermodynamic equilibrium is an accurate measure of the chemical potential of ethanol in the water phase. Therefore headspace GC is a convenient method for measurement of ethanol concentration in solution. This idea has been put to practical use in the routine determination of blood alcohol concentrations by headspace GC. For measurement of ethanol partitioning into membranes, vapor pressure should be ideally measured directly, without the addition of substances that serve as internal calibration standards. All calibration substances we have encountered exhibit varying degrees of partitioning into membranes thereby confounding the measurement of ethanol vapor pressure. We established conditions that avoid instrument drift and ran calibration samples intermittently between membrane samples. This yielded experimental conditions that permit ethanol vapor pressure measurements within $\pm 1 \%$ error under most circumstances, allowing for very precise measurements of free ethanol concentration in the excess water phase. The difference in free ethanol concentrations between samples with and without added membranes is a precise measure of ethanol partitioning into the lipid matrix. The method does not require separation of membranes and water by centrifugation which greatly simplifies matters compared to analogous studies using radioisotopes.

The method has sufficient sensitivity for measurement at physiological concentrations of lipids and ethanol. That is, 20 wt $\%$ lipid, $80 \%$ water with an initial ethanol concentration of $20 \mathrm{mmol} / \mathrm{l}$. The lipid headgroup, backbone, and acyl chain composition was varied. The data indicate that ethanol partitions primarily into the lipid/water interface of membranes and that chemical modification of the membrane interface alters partition coefficients.

\section{Materials and Methods}

The lipids 1-palmitoyl-2-oleoyl-sn-glycero-3-phosphocholine (POPC), 1,2-dinervonyl-sn-glycero-3-phosphocholine (DNPC), 1,2-dilauroyl-sn-glycero-3-phosphocholine (DLPC), 1-palmitoyl-2-oleoyl-sn-glycero-3-phosphoethanolamine (POPE), 1-palmitoyl-2-oleoyl-sn-glycero-3phosphoserine (POPS), 1-palmitoyl-2-oleoyl-sn-glycero-3[phospho-rac-(1-glycerol)] (POPG), soybean phosphatidylinositol (PI) mixture, and brain sphingomyelin mixture (SM) were purchased from Avanti Polar Lipids, Inc. (Alabaster, Alabama, U.S.A.). Bovine brain ganglioside mixture was purchased from Calbiochem (La Jolla, California, U.S.A.). The 200 proof ethanol was purchased from The WarnerGraham Company (Cockeysville, Maryland, U.S.A.).

Samples containing $20 \mathrm{wt} \%$ of membranes were prepared by weighing $50 \mathrm{mg}$ of lipid into a headspace vial and adding $200 \mu \mathrm{l}$ of $20 \mathrm{mmol} / \mathrm{l}$ ethanol solution to bring the total mass to $250 \mathrm{mg}$. Control vials contained $200 \mu \mathrm{l}$ of ethanol solution. A blank, consisting of the lipid plus $200 \mu \mathrm{l}$ of water was run in series with each experiment to ensure that samples did not release any vapors that could contribute to the ethanol peak in the chromatogram. For lipid mixtures, the components were weighed and dissolved in warm $2: 1$ chloroform/methanol solution and dried under an argon stream to a thin film. The film was taken up in $500 \mu \mathrm{l}$ of water, frozen, and dried under vacuum for $24 \mathrm{~h}$. The vials were reweighed and the ethanol solution was added. Vials were then sealed and put through seven repetitive cycles of freezing, thawing and vortexing to ensure homogenous distribution of water and ethanol.

\section{Headspace gas chromatography}

Measurements of ethanol concentration in the headspace were performed using a HP 6890 series gas chromatograph equipped with a flame ionization detector. A $30 \mathrm{~m} \times 0.53 \mathrm{~mm}$ $\times 40 \mu \mathrm{m}$ HP-PLOT Q polystyrene-divinyl-benzene-based column was utilized in order to resolve ethanol from various short-chain hydrocarbon gases that can arise from residual solvent and traces of oxidation in the samples. A HP 7694 headspace sampler with $1 \mathrm{ml}$ sample loop and silicosteel transfer line was connected directly to the split inlet of the GC (all Hewlett-Packard, Wilmington, Delaware, U.S.A.).

Operating conditions were as followed: headspace sampler oven, loop, and transfer line temperatures were 50, 80, and $80^{\circ} \mathrm{C}$, respectively; time parameters for vial equilibration, vial pressurization, loop fill, loop equilibration, injection time, and GC cycle time were 60, 0.15, 0.15, 0.15, 0.5, and $10 \mathrm{~min}$, respectively. Because no internal standard was used, additional precautions were required to ensure reproducibility of headspace injection volume and to avoid condensation of vapors. This was achieved through insulation of exposed areas between sampler and GC inlet to minimize temperature gradients, maintaining an isothermal oven temperature of $140^{\circ} \mathrm{C}$, and preconditioning the system with injections of similar ethanol concentration for at least six hours prior to running samples in order to achieve steady-state conditions. Headspace vials were continuously shaken during equilibration, and pressurized with $22.0 \mathrm{psi}$ of helium. The inlet temperature was maintained at $250^{\circ} \mathrm{C}$ with a split ratio of $2: 1$. Helium was used as the carrier gas with a flow rate of $6.0 \mathrm{ml} / \mathrm{min}$. Data acquisition and peak integration was performed using HP Chemstation software (Hewlett-Packard). Samples with a known ethanol concentration were run 
intermittently with lipid samples to detect and compensate for any instrument drift. Typically five samples were prepared for every data point and results averaged.

\section{Results and Discussion}

Fig. 1 shows the area of the ethanol peak in the GC chromatograms vs. ethanol concentration obtained on a series of test samples with known ethanol content. Linearity was excellent, permitting measurements of ethanol concentration to $\pm 0.2 \mathrm{mmol} / \mathrm{l}$ over the concentration range of $2-20 \mathrm{mmol} / \mathrm{l}$. Measurements were conducted at a sample temperature of $50^{\circ} \mathrm{C}$ where reproducibility was optimal.

Ethanol partitioning into POPC bilayers was studied by adding POPC to a solution of $20 \mathrm{mmol} / \mathrm{l}$ ethanol in water. Experiments were conducted over the POPC concentration range of $0-40 \mathrm{wt} \%$. Results are presented in Fig. 2. Ethanol vapor pressure in the headspace above samples decreases with increasing lipid concentration. The decrease is caused by partitioning of ethanol into POPC bilayers. The membranes and the excess water/ethanol solution represent separate phases. In thermodynamic equilibrium, the chemical potential of ethanol in the vapor above the sample, in the water phase, and in the lipid bilayer phase are equal. Therefore the ethanol vapor pressure in the headspace is an accurate measure of ethanol concentration in the water phase. With a calibration curve as shown in Fig. 1, the ethanol concentration in the water phase was determined. The reduction of ethanol concentration from the initial value of $20 \mathrm{mmol} / \mathrm{l}$ in the water phase yields the amount of ethanol in the lipid

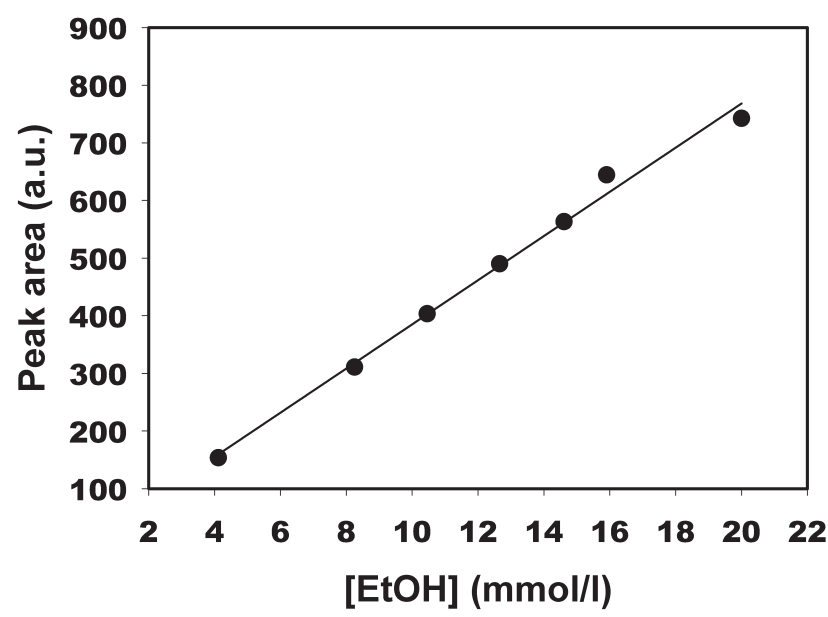

Figure 1. Ethanol peak area of the headspace gas chromatogram as a function of ethanol concentration in solution showing excellent linearity of measurements. Calibration samples were run intermittently between membrane samples to monitor performance of the equipment.

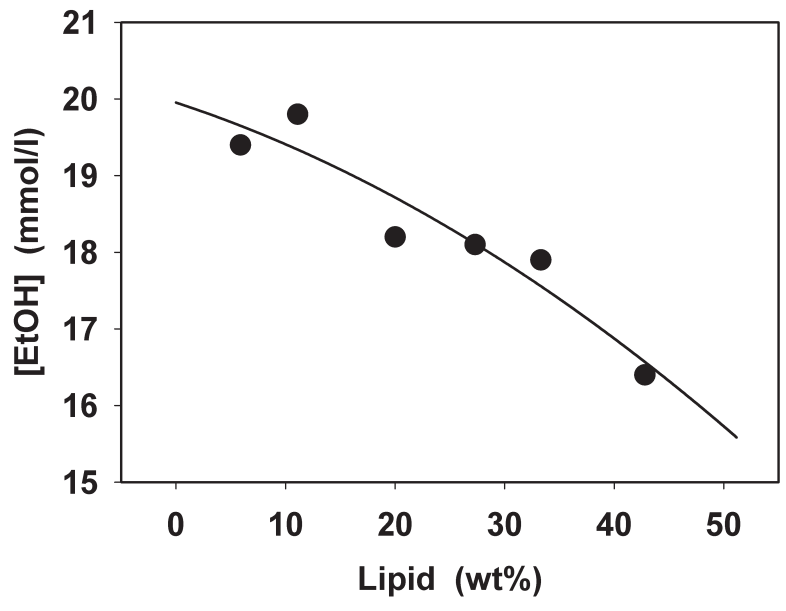

Figure 2. Ethanol concentration in solution as a function of POPC concentration in the lipid/water dispersion. The solid line is a fit to the experimental data points assuming a molar partition coefficient of $K_{p}=19$.

phase. The molar coefficient of ethanol partitioning into lipid bilayers, $K_{p}$ is defined as

$$
K_{p}=\frac{X_{\text {lipid }}^{E t O H}}{X_{\text {water }}^{\text {EtOH }}}
$$

where $X_{\text {lipid }}^{E t O H}$ is the mole fraction of ethanol in the lipid phase and $X_{\text {water }}^{\mathrm{EtOH}}$ the mole fraction of ethanol in the water phase. A small fraction of added water becomes part of the lipid phase ( Katz and Diamond 1974b; Gawrisch et al. 1985). This bound water raises the interfacial volume of lipids but is not considered in the calculation of the ethanol mole fraction in the lipid phase. The reduction of the volume of the water phase from lipid hydration has some influence on the calculation of partition coefficients. Balgavy and co-workers determined the number of water molecules per phosphatidylcholine intercalated into the bilayer polar region by small angle neutron scattering and obtained a value of $7 \pm 2$ waters (Balgavy et al. 2001). This value was used for the calculation of partition coefficients of all investigated lipids. While this assumption is crude, it is unlikely to alter principle conclusions. A fit of the data in Fig. 2 yielded a molar partition coefficient of ethanol into POPC bilayers of $K_{p}=19$ (solid line). This value is slightly lower than ethanol partitioning into 1,2-dimyristoyl-sn-glycero-3-phosphocholine bilayers reported by Katz and Diamond (1974c) obtained by the radioisotope method and Trandum et al. (1999) by calorimetry, but significantly higher than partition coefficients obtained by Rowe using calorimetry (Rowe 1981). Please note that in some of those papers partition coefficients are 
Table 1. Ethanol concentration in bulk water $[\mathrm{EtOH}]_{\text {bulk }}$ after equilibration of a $20 \mathrm{mmol} / \mathrm{l}$ ethanol solution with a $20 \mathrm{wt} \%$ lipid dispersion. From the decline of ethanol concentration in the water phase a molar ethanol partition coefficient $K_{p}$, the interfacial ethanol concentration $[\mathrm{EtOH}]_{\text {interf }}$, the lipid/ethanol molar ratio, and the percentage of total ethanol that is located in the lipid/water interface were calculated (see text for details). Experiments were conducted at $50^{\circ} \mathrm{C}$

\begin{tabular}{|l|c|c|c|c|c|}
\hline & $\begin{array}{c}{[\mathrm{EtOH}]_{\text {bulk }}} \\
(\mathrm{mmol} / \mathrm{l})\end{array}$ & $K_{p}$ & $\begin{array}{c}{[\mathrm{EtOH}]_{\text {interf }}} \\
(\mathrm{mmol} / \mathrm{l})\end{array}$ & $\begin{array}{c}\text { Lipid/ethanol } \\
(\mathrm{mol} / \mathrm{mol})\end{array}$ & \% bound \\
\hline POPG & $17.6 \pm 0.4$ & $30 \pm 5$ & $27 \pm 4$ & $104 \pm 15$ & $15.6 \pm 2.0$ \\
\hline POPS & $17.8 \pm 0.3$ & $28 \pm 3$ & $25 \pm 3$ & $109 \pm 12$ & $14.6 \pm 1.5$ \\
\hline POPC/SM $(1: 1)^{\mathrm{a}}$ & $17.6 \pm 0.2$ & $29 \pm 2$ & $29 \pm 2$ & $107 \pm 6$ & $15.7 \pm 1.1$ \\
\hline PI & $18.3 \pm 0.2$ & $24 \pm 2$ & $18 \pm 2$ & $123 \pm 11$ & $11.9 \pm 0.9$ \\
\hline POPC & $18.6 \pm 0.3$ & $19 \pm 2$ & $19 \pm 2$ & $151 \pm 23$ & $10.8 \pm 1.4$ \\
\hline POPC/gang $(9: 1)^{\mathrm{b}}$ & $18.8 \pm 0.9$ & $19 \pm 9$ & $15 \pm 7$ & $156 \pm 85$ & $9.5 \pm 4.3$ \\
\hline DLPC & $18.7 \pm 0.3$ & $16 \pm 2$ & $16 \pm 2$ & $179 \pm 26$ & $11.2 \pm 1.4$ \\
\hline DNPC & $19.3 \pm 0.4$ & $15 \pm 5$ & $16 \pm 4$ & $195 \pm 60$ & $6.7 \pm 2.0$ \\
\hline POPE & $19.1 \pm 0.3$ & $14 \pm 2$ & $17 \pm 3$ & $200 \pm 40$ & $8.7 \pm 1.5$ \\
\hline
\end{tabular}

${ }^{a}$ mixture of POPC and SM at a weight ratio of $1: 1 ;{ }^{b}$ mixture of POPC and gang (ganglioside) at a weight ratio of $9: 1$.

reported as a quotient of ethanol concentrations in the membrane and water phase. The concentration of ethanol is calculated as moles of ethanol per unit of mass of membrane and water, respectively. Partition coefficients calculated in this fashion are numerically different from molar partition coefficients.

Experiments on a series of lipids and lipid mixtures with different headgroups and a sphingosine backbone instead of glycerol were conducted at the fixed lipid concentration of $20 \mathrm{wt} \%$. Results are summarized in Table 1. The lowering of ethanol concentration from the initial value of 20 $\mathrm{mmol} / \mathrm{l}$ is a good measure of partitioning into the bilayers. Highest partition coefficients were observed for POPG, POPC + $50 \mathrm{wt} \%$ sphingomyelin, and POPS. The lipids PI, POPC and POPC containing $10 \mathrm{wt} \%$ brain gangliosides showed somewhat lower ethanol partitioning. The lipids DLPC with 12 carbon atoms per hydrocarbon chain and DNPC with 24 carbon atoms per chain and POPE had the lowest partition coefficients. Clearly, the twofold changes of ethanol partition coefficients over the series of investigated lipids are caused by differences in polar interactions between lipids and ethanol.

It is remarkable that the almost twofold difference in hydrophobic volumes between DLPC with 12 carbon atoms per hydrocarbon chain and DNPC with 24 atoms per chain did not yield any difference in ethanol partitioning. The results indicate that ethanol partitions into the lipid/water interface of bilayers while interaction with the hydrophobic core is unimportant. It confirms our previous results on the location of ethanol in POPC bilayers obtained by NOESY (nuclear Overhauser enhancement spectroscopy) NMR and molecular simulations (Feller et al. 2002). These experiments showed that ethanol resides preferentially in the region of phosphate groups of phospholipids where it engages in hydrogen bonding. Ethanol concentration declines steeply over the region of carbonyl groups of lipid hydrocarbon chains towards the bilayer center, with frequent incursions of ethanol molecules into the region of upper hydrocarbon chain segments. The lifetimes of association between lipid and ethanol are brief, on the order of nanoseconds only (Koenig and Gawrisch 2005a). Ethanol

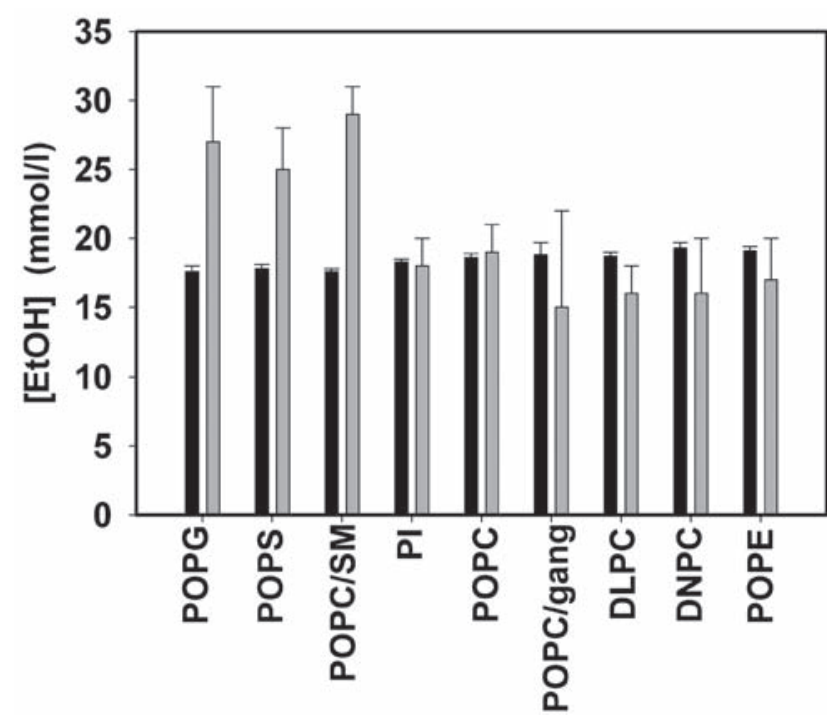

Figure 3. Ethanol concentration in the excess water phase (black bars) and ethanol concentration in the lipid/water interface (grey bars) for the investigated lipid/water dispersions calculated with the assumption that ethanol partitions into the lipid/water interface comprised of lipid headgroups, intercalated water, glycerol or sphingosine backbones, and carbonyl groups of lipid hydrocarbon chains. 
is in rapid exchange between the excess water phase and the lipid water interface.

With the assumption that ethanol concentration in the hydrophobic core of bilayers is negligible, the ethanol concentration in the lipid/water interface can be calculated. The volumes of hydrocarbon chain segments are $\mathrm{V}_{\mathrm{CH} 3}=54 \AA^{3}$, $\mathrm{V}_{\mathrm{CH} 2}=27 \AA^{3}$, and $\mathrm{V}_{\mathrm{CH}}=20.5 \AA^{3}$ (Tardieu et al. 1973). Lipid density is close to $1 \mathrm{~g} / \mathrm{cm}^{3}$ (Koenig and Gawrisch 2005b). The latter yields a volume of lipid molecules from which the volume of the hydrophobic chain segments is subtracted to yield an interface volume. It includes the carbonyl groups of lipid hydrocarbon chains, the glycerol or sphingosine groups, and the lipid headgroup. The volume of intercalated water was added assuming a volume of $30 \AA^{3}$ per water molecule. The amount of ethanol that is lipid-bound is divided by this volume to yield an ethanol concentration in the lipid/water interface. Corresponding interfacial ethanol concentrations are reported in Table 1 and shown in Fig. 3. Ethanol concentrations in the water phase and the lipid/water interface are comparable, with somewhat higher values for ethanol in the interface of POPG, POPS, and POPC/SM bilayers.

For practical considerations, the number of ethanol molecules per lipid in the lipid/water interface is important. At the physiological ethanol concentration of $20 \mathrm{mmol} / \mathrm{l}$, just one ethanol molecule binds to 100-200 lipids (see Table 1). The fraction of ethanol in the lipid/water interface is $6-16 \%$ of total ethanol in the sample. The low probability of ethanol associating with lipids at $20 \mathrm{mmol} / \mathrm{l}$ ethanol explains why changes of lipid order are not detectable at such low ethanol content. In contrast, changes of lipid order at molar ethanol concentrations are substantial (Barry and Gawrisch 1994, 1995). At those concentrations, on average, one ethanol molecule is associated with every lipid. This raises questions about the importance of ethanol binding to lipid bilayers. While binding events of ethanol to lipids at physiological concentrations of ethanol are rare, it has to be considered that membrane proteins in the lipid matrix interact with an entire domain of lipids. The interfacial interaction of ethanol with those lipid domains may shift the energetics of lipid-protein interaction sufficiently to cause a shift in protein function. It is also possible that the lipid-protein interface has particular affinity for ethanol.

\section{Conclusions}

Ethanol binding to lipid bilayers by headspace GC is a convenient tool to study interaction of ethanol with biopolymers. Compared to other methods, the partial ethanol vapor pressure measurement excels in precision and ease of application. Comparison of ethanol partition coefficients between membranes with different lipid headgroups indi- cated that properties of the lipid/water interface are critical for ethanol partitioning while the volume of the hydrophobic phase is unimportant. We propose a layer model of ethanol partitioning into lipid membranes, with ethanol residing in the lipid/water interface, and with negligible ethanol concentrations in the bilayer hydrophobic core. We calculated ethanol concentrations for the lipid/water interface and found them comparable to ethanol concentrations in the bulk solution. Ethanol may alter membrane protein function through its influence on interfacial energies of lipids and proteins.

Acknowledgement. This work was supported by the Intramural Research Program of NIAAA, NIH.

\section{References}

Balgavy P., Dubnickova M., Kucerka N., Kiselev M. A., Yaradaikin S. P., Uhrikova D. (2001): Bilayer thickness and lipid interface area in unilamellar extruded 1,2-diacylphosphatidylcholine liposomes: a small-angle neutron scattering study. Biochim. Biophys. Acta 1512, 40-52; doi:10.1016/S0005-2736(01)00298-X

Barry J. A., Gawrisch K. (1994): Direct NMR evidence for ethanol binding to the lipid-water interface of phospholipid bilayers. Biochemistry 33, 8082-8088; doi:10.1021/ bi00192a013 PMid:8025114

Barry J. A., Gawrisch K. (1995): Effects of ethanol on lipid bilayers containing cholesterol, gangliosides, and sphingomyelin. Biochemistry 34, 8852-8860; doi:10.1021/bi00027a037 PMid:7612626

Chin J. H., Goldstein D. B. (1977): Drug tolerance in biomembranes: a spin label study of the effects of ethanol. Science 196, 684-685; doi:10.1126/science.193186 PMid:193186

Feller S. E., Brown C. A., Nizza D. T., Gawrisch K. (2002): Nuclear Overhauser enhancement spectroscopy cross-relaxation rates and ethanol distribution across membranes. Biophys. J. 82, 1396-1404; doi:10.1016/S0006-3495(02)75494-5 PMid:11867455 PMCid:1301941

Franks N. P., Lieb W. R. (1984): Do general anesthetics act by competitive binding to specific receptors? Nature 310, 599-601; doi:10.1038/310599a0 PMid:6462249

Gawrisch K., Richter W., Mops A., Balgavy P., Arnold K., Klose G. (1985): The influence of water concentration on the structure of egg-yolk phospholipid water dispersions. Studia Biophys. 108, 5-16

Katz Y., Diamond J. M. (1974a): Method for measuring nonelectrolyte partition coefficients between liposomes and water. J. Membr. Biol. 17, 69-86; doi:10.1007/BF01870173 PMid:4600821

Katz Y., Diamond J. M. (1974b): Nonsolvent water in liposomes. J. Membr. Biol. 17, 87-100; doi:10.1007/BF01870174 PMid:4858130

Katz Y., Diamond J. M. (1974c): Thermodynamic constants for nonelectrolyte partition between dimyristoyl lecithin 
and water. J. Membr. Biol. 17, 101-120; doi:10.1007/ BF01870175 PMid:4407659

Koenig B. W., Gawrisch K. (2005a): Lipid-ethanol interaction studied by NMR on bicelles. J. Phys. Chem. B 109, 7540-7547; doi:10.1021/jp044980b PMid:16851866

Koenig B. W., Gawrisch K. (2005b): Specific volume of unsaturated phosphatidylcholines in the liquid crystalline phase. Biochim. Biophys. Acta 1715, 65-70; doi:10.1016/ j.bbamem.2005.07.006

Rowe E. S. (1981): Membrane: buffer partition coefficient for ethanol in dimyristoylphosphatidylcholine. Alcohol. Clin. Exp. Res. 5, 259-263; doi:10.1111/j.1530-0277.1981. tb04899.x

Rowe E. S. (1982): The effects of ethanol on the thermotropic properties of dipalmitoylphosphatidylcholine. Mol. Pharmacol. 22, 133-139

Tardieu A., Luzzati V., Reman F. C. (1973): Structure and polymorphism of the hydrocarbon chains of lipids: a study of lecithin-water phases. J. Mol. Biol. 75, 711-733; doi:10.1016/0022-2836(73)90303-3 PMid:4738730

Trandum C., Westh P., Jorgensen K., Mouritsen O. G. (1999): Association of ethanol with lipid membranes containing cholesterol, sphingomyelin and ganglioside: a titration calorimetry study. Biochim. Biophys. Acta 1420, 179-188; doi:10.1016/S0005-2736(99)00092-9

Westerman P. W., Pope J. M., Phonphok N., Doane J. W., Dubro D. W. (1988): The interaction of $n$-alkanols with lipid bilayer membranes: a 2H NMR study. Biochim. Biophys. Acta 939, 64-78; doi:10.1016/0005-2736(88)90048-X

Westh P., Trandum C. (1999): Thermodynamics of alcohol-lipid bilayer interactions: application of a binding model. Biochim. Biophys. Acta 1421, 261-272; doi:10.1016/S00052736(99)00130-3

Received: December 9, 2008

Final version accepted: February 20, 2009 